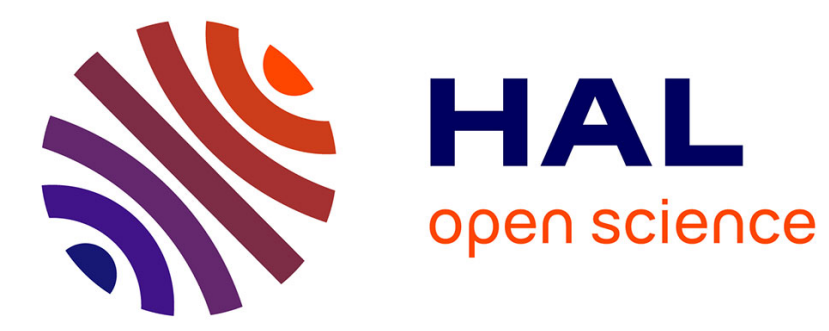

\title{
Le thème épigraphique de la porte
}

Robert Favreau

\section{- To cite this version:}

Robert Favreau. Le thème épigraphique de la porte. Cahiers de Civilisation Médiévale, 1991, 34, pp.267-279. 10.3406/ccmed.1991.2499 . halshs-03203009

\section{HAL Id: halshs-03203009 \\ https://shs.hal.science/halshs-03203009}

Submitted on 20 Apr 2021

HAL is a multi-disciplinary open access archive for the deposit and dissemination of scientific research documents, whether they are published or not. The documents may come from teaching and research institutions in France or abroad, or from public or private research centers.
L'archive ouverte pluridisciplinaire HAL, est destinée au dépôt et à la diffusion de documents scientifiques de niveau recherche, publiés ou non, émanant des établissements d'enseignement et de recherche français ou étrangers, des laboratoires publics ou privés. 


\section{Le thème épigraphique de la porte}

\section{Robert Favreau}

\section{Citer ce document / Cite this document :}

Favreau Robert. Le thème épigraphique de la porte. In: Cahiers de civilisation médiévale, $34 \mathrm{e}$ année ( $\left.{ }^{\circ} 135-136\right)$, Juilletdécembre 1991. La façade romane. Actes du Colloque international organisé par le Centre d'Etudes Supérieures de Civilisation Médiévale. Poitiers, 26-29 septembre 1990. pp. 267-279;

doi : https://doi.org/10.3406/ccmed.1991.2499

https://www.persee.fr/doc/ccmed_0007-9731_1991_num_34_135_2499

Fichier pdf généré le 25/03/2019 


\begin{abstract}
The doorway was a privileged meeting place in the Old Testament, as it was throughout the Middle Ages. It was used to show off inscriptions destined to a widest public. As the entrance to the church, it received special treatment. The inscriptions reveal the patrons.

The doorway opening into the " House of God " is the "Gate of Heaven". Inscriptions are drawn from Jacob's vision (Gn, 28, 10-22) and from the consecration liturgy. The church is also the " House of Prayer ", a place of peace where the faithful are invited to do penitence.

But above all, the doorway is Christ himself, an idea taken up in numerous inscriptions. The Virgin, a gate opened for the advent of Christ but then closed, is also the " Gate of Heaven ".
\end{abstract}

\title{
Résumé
}

La porte est un lieu privilégié de rencontre dans l'Ancien Testament et tout au long du moyen âge. servi de support aux inscriptions dont on voulait assurer la meilleure publicité. Entrée de l'église, elle fait l'objet de grands soins et des inscriptions en rappellent les commanditaires.

La porte de l'église ouvre sur la « maison de Dieu » qui est « porte du ciel ». Les inscriptions s'inspirent ici de la vision de Jacob (Gn, 28, 10-22) et de la liturgie de dédicace.

L'église est « maison de prière » et lieu de paix pour le fidèle, invité à y entrer avec des sentiments de pénitence.

Surtout la porte est le Christ lui-même, ce que reprennent de nombreuses inscriptions. Marie, porte ouverte pour la venue du Christ mais ensuite fermée, est aussi « porte du ciel ». 


\section{${ }^{*}$ Robert FAVREAU}

\section{Le thème épigraphique de la porte}

\section{RESIME}

La porte est un lieu privilégié de rencontre dans l'Ancien Testament et tout au long du moyen àge. Elle a servi de support aux inscriptions dont on voulait assurer la meilleure publicité. Entrée de l'église, elle fait l'objet de grands soins et des inscriptions en rappellent les commanditaires.

La porte de l'église ouvre sur la "maison de Dieu" qui est "porte du ciel». Les inscriptions s"inspirent ici de la vision de Jacob ( $\mathrm{Gn}, 28,10-22)$ et de la liturgie de dédicace.

l'église est "maison de prière" et lieu de paix pour le fidèle, invitè à y entrer avec des sentiments de pénitence.

Surtout la porte est le Christ lui-même, ce que reprennent de nombreuses inscriptions. Marie, porte ouverte pour la venue du Christ mais ensuite fermée, est aussi "porte du ciel".

The doorway was a privileged meeting place in the Old Testament, as it was throughout the Middle Ages. It was used to show off inscriptions destined to a widest public. As the entrance to the church, it received special treatment. The inscriptions reveal the patrons.

The doorway opening into the "House of God" is the "Gate of Heaven". Inscriptions are drawn from Jacob's vision (Gn, 28, 10-22) and from the consecration liturgy. The church is also the "House of Prayer", a place of peace where the faithful are invited to do penitence.

But above all, the doorway is Christ himself, an idea taken up in numerous inscriptions. The Virgin, a gate opened for the advent of Christ but then closed, is also the "Gate of Heaven".

Le mot "porte" est mentionné plus de trois cents fois dans l'Ancien Testament, le plus souvent pour désigner la porte d'une ville. La porte fortifiée assure la sécurité des habitants de la cité. L'espace libre devant la porte sert de place publique. Notables, vieillards, jeunes s'y retrouvent ${ }^{1}$. On y passe des transactions commerciales ${ }^{2}$, et surtout on y rend des jugements ${ }^{3}$. Les rois d'Israël et de Juda y siègent en grand apparat avant de partir en campagne ${ }^{4}$. Au moyen âge l'église est le lieu par excellence des rassemblements de la communauté. Devant ses portes des parties du culte se déroulent, telles que dédicace, baptême, mariage, processions. Parfois on y prèche, on y fait les annonces publiques, le marché s'y tient. La place devant le porche de l'église est donc un lieu public important. On voit bien l'efficacité de la publicité donnée aux privilèges concédés par l'empereur Henri V en 1111 aux habitants de Spire, dès lors qu'on les avait gravés en lettres d'or sur les portes de la cathédrale, comme aux privilèges donnés à Mayence par l'archevêque Adalbert. en 1135 à partir du moment où on pouvait les lire sur la porte de bronze de la cathédrale, et on

1. Job. 29, 7-9; - Ps. 69 (68). 13, qui sedebant in porta.

2. Genèse, $23,10-18$.

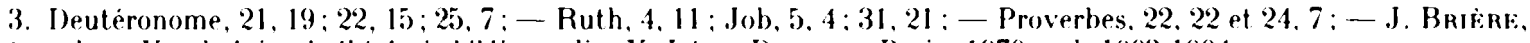
"Porte", dans Vocabulaire de theologie biblique, dir. X. LÉn-DCFolr, Paris, 1970, col. 1(0)3-10(0)4.

4. 1 Rois. 22.10. 
fixera encore sur la porte de la cathédrale de Worms une inscription rappelant une franchise accordée à la ville par l'empereur Frédéric Ier ${ }^{5}$. La porte de l'église se prête particulièrement bien à la délivrance d'un message, et l'on sait la richesse des programmes iconographiques, commentés par des inscriptions, qui se développent sur les portes de Sainte-Sabine de Rome, des cathédrales de Mayence, Hildesheim, Novgorod, du Puy, ou des églises de Saint-Paul-hors-les-murs à Rome, de Monte Sant'Angelo, etc. ${ }^{6}$.

Entrée de l'église, la porte mérite, en elle-même, le meilleur traitement, et une série d'inscriptions y est tracée pour indiquer le patronage de l'édifice, Jean-Baptiste à l'oratoire près du baptistère du Latran, Paul à Saint-Paul-hors-les-murs, Michel à Monte Sant'Angelo, Dieu et Marie à Ravello, le nom de l'auteur de la porte, Bérenger à Mayence vers l'an mil, Roger d'Amalfi à S. Sabino de Canosa ${ }^{7}$, ou surtout pour mettre à l'honneur le donateur. Le pape Hilaire (461-468) offre les vantaux de bronze de l'oratoire Saint-Jean-Baptiste au Latran ${ }^{8}$, l'inscription des portes de bronze de Sainte-Sophie de Constantinople nomme les empereurs Théophile et Michel en $838^{9}$, l'archevêque de Mayence Willigis (975-1011) fait faire la porte nord de sa cathédrale ${ }^{10}$. Les inscriptions deviennent ensuite peu à peu plus explicites. Quand il fait placer des portes de bronze au portail ouest de sa cathédrale d'Hildesheim, Bernward indique expressément qu'il escompte qu'on se souviendra de lui en voyant ces portes placées à "la façade du temple angélique» ". Poussé par l'amour de saint Paul, le consul d'Amalfi, Pantaléon, a commandé à Constantinople, en 1070, la confection des portes de bronze de Saint-Paul-hors-les-murs; il demande à quiconque entrera dans la basilique de regarder ces portes avec le plus grand soin, et, une fois entré, de prier Dieu d'accorder le repos éternel à celui qui a fait un don aussi remarquable ${ }^{12}$. En 1076, la porte de bronze de Monte Sant'Angelo, commandée par un autre Pantaléon, est achevée à Constantinople. Sur le battant de gauche Pantaléon prie le clergé du sanctuaire de faire nettoyer ces portes une fois l'an, afin qu'elles soient toujours brillantes et éclatantes. Sur le vantail de droite il demande à quiconque viendra prier en cette église "d'admirer d'abord un si bel ouvrage", puis, une fois entré, de prier pour l'âme de l'"auteur de ce travail», c'est-à-dire pour lui-même; suit une prière à saint Michel pour qu'il écoute les prières en faveur de Pantaléon qui a fait si bien décorer le sanctuaire qui lui est consacré ${ }^{13}$. Comme à Saint-Paul-hors-les-murs il s'agit encore des portes matérielles, mais on voit déjà qu'elles tirent leur importance du fait qu'elles ouvrent sur une maison "de prière".

5. Robert Favreav, "Fonctions des inscriptions au moyen âge", Cahiers de Civilisation médiëvale, XXXII, 1989. p. 211.

6. Ursula Mende, Albert Hirmfr et Irmgard Eanstmeifr-Hirmer, Die Bronzetüren des Millelallers 800-1200, Munich, 1983: - Ilermann Lfisinger, Romanische Bronzen. Kirchentüren in miltelaterlichen Europa, Zurich. 1956: (Gisela Jerramas, Die Holztür der Basilika S. Sabina in Rom ..., Tübingen, 1980 ("Bilderhefte d. deutschen archäolog. Instit. Rom," 7).

7. Les textes sont donnés dans L. Mende, A. Hirmer et I. Ennstmfier-IIrmer, Die Bronzelüren ...

8. H. Leci.facQ, "Porte", dans Dictionnaire d'archéologie chrétienne el de lilurgie, XIV-1, Paris, 1939, col. 1506; Philippe Lacfr, Le palais de Latran. Étude historique et archéologique, Paris, 1911, p. 53-54.

9. H. Lecifreq. "Porte", art. cit., col. 1507. RAT.

10. MFxDe, op. cit. p. 133-134 : WILLIGISUS ARCHIEPISCOPUS EX METALLI SPECIE VALVAS EFFECE-

11. Mende, op. cit., p. 135-136: - Wilhelm Brages, Die älteren Hildesheimer Inschriften bis zum Tode Hezilos (†1079), Göttingen, 1983. p. 109-116, pl. 20 ("Abhand. d. Akad. d. Wissensch. zu Göttingen. Phil. hist. Kl.". 131) : A.V.NO DOMINICE INCARNATIONIS MXV BER.NWARDUS EPISCOPLS DIVE MEMORIE HAS VALVAS FUSILES IN FACIEM A.NGELICI TEMPLI OB MONIMENTUM SLI FECIT SUSPE.NII.

12. Vincenzo Forcer.ta, Iscrizioni delle chiese e d'altri edificii di Roma dal secolo XI fino ai giorni nostri. XII, Rome. 1878, no 5, p. 10, no 6, p. 11; - Horazio Marccchi, Basiliques el églises de Rome, Paris/Rome, 1902, p. 145; Guglielmo Mattriaf, Le porte bronzee bizantine in Italia, Rome, 1971, p. 81-82. Le mot portae est cité trois fois.

13. (;. Matrhiaf, op. ril. p. 83; - Alfredo Petrucci, Caltedrali di Puglia. 2e éd., Rome. 1965. fig. 11-12: RoGO) VOS OMNES QUI HIC VE.NITIS CALSA ORATIONIS, UT PRIUS INSPICIATIS TAM PULCIIRUM LABOREM, ET SIC INTRANTES PRECAMINI DOMINUM PRONI PRO ANIMA PANTALEONIS QUI FUIT AITOR HUJIS I.ABORIS. 
Sur la porte sud de la cathédrale de Troia l'évêque Guillaume a tenu, en 1127, à faire indiquer qu'il a payé le travail de ses propres ressources ${ }^{14}$, et au milieu du xir $^{\mathrm{e}} \mathrm{s}$. l'évêque Alexandre de Plock se fait représenter, avec son nom, au portail ouest de la cathédrale de Novgorod ${ }^{15}$. Sur les portes occidentales de la cathédrale de Ravello, en 1179, Serge Mussetula, qui a fait faire le travail, demande que l'on se souvienne de lui, de sa femme, de leurs deux fils et de leur fille, avec la formule même du canon de la messe, MEMENTO, DOMINE, FAMULI TLI ... ${ }^{16}$. Plusieurs inscriptions sont plus précises : un don aussi précieux qu'une porte mérite à son auteur que la porte du ciel lui soit ouverte. Au linteau de la porte principale de San Marcello de Capoue, une inscription du début du XII" s. nous dit que "l'abbé Alferius donne au Christ ces blanches portes, afin de mériter d'entrer dans le royaume des cieux " ${ }^{17}$, et au-dessus de la porte gauche de la cathédrale de Barletta on lit que "Richard a fait faire à ses dépens cette porte brillante, ce qui lui méritera de voir la porte du ciel s'ouvrir pour lui dans la joie»" ${ }^{18}$.

En fait le grand nombre des inscriptions qui traitent de la porte nous invite à ne pas rester à la seule beauté matérielle de la porte, si grande soit-elle. A Saint-Denis, peu avant le milieu du $\mathrm{XII}^{\mathrm{e}}$ s., l'abbé Suger fait faire, par des fondeurs et des sculpteurs soigneusement choisis, les portes principales de son église, où sont représentées la Passion, la Résurrection et l'Ascension du Seigneur. Il y fait inscrire, en lettres de cuivre dorées huit vers qui traduisent son souci de voir le fidèle passer de la forme extérieure au sens spirituel : il ne s'agit pas de s'extasier sur la beauté de ces portes, mais d'être conduit, par la perfection de l'ouvrage, vers la "vraie lumière dont le Christ est la vraie porte. La porte dorée invite à connaître ce qui est à l'intérieur. L'esprit engourdi s'élève vers le vrai par les choses matérielles", MENS HEBES AD VERUM PER MATERIALIA SURGIT ${ }^{19}$, phrase forte qui renvoie directement au Christ-Dieu, Deum verum de Deo vero du Credo, et à la Résurrection, le verbe surgere étant employé dans le Nouveau Testament pour la Résurrection du Christ et les miracles par lui opérés. L'inscription du linteau, à la façade de la cathédrale de Troia, renvoie expressément du "matériel" au "spirituel» :

\section{+ ISTIUS AECCLESIAE PER PORTAM MATERIAI.IS \\ INTROITUS NOBIS TRIBUATUR SPIRITUALIS,}

"par la porte de cette église matérielle, une entrée spirituelle nous est offerte»"20.

C'est qu'en effet il ne s'agit pas d'une porte quelconque mais de la porte d'une église. Déjà sous l'Ancien Testament les portes des fils du peuple élu étaient-elles distinguées des autres : pour que l'ange du Seigneur épargne les Israélites en terre d'Égypte, ceux-ci avaient dû immoler un agneau et marquer de son sang le linteau et les deux montants de porte de leur maison ${ }^{21}$, et par la bouche de Moïse le Seigneur avait prescrit à Israël de garder dans son cœur ses commandements et de «les

14. MENDE, op. cit., p. 144

15. Ibid., p. 156.

16. Ibid., p. 165-166.

17. Mario I'(ONofrio et Valentino Pacr, Campanie romane, La Pierre-qui-Vire, 1981, p. 42.

18. Centre d'études supérieures de civilisation médiévale, photothèque:

IMPENSIS, RICHARDE, TLIS IIEC PORTA NITEBIT.

ER(;O) TIBI. MERITO, CELESTIS IETA PATEBIT

19. Sugre, (Euvres completes, éd. A. Lecoy df La Marchf, Paris, 1867, p. 189; - Philipe Vramifr, "Saint-Denis et la tradition carolingienne des tituli. Le le rebus in administratione sua geslis de Suger", dans La Chanson de geste el le mylhe carolingien. Mélanges René Locis. I, Saint-Père-sous-Vèzelay, 1982, p. 342-343.

20. Mario I) SAvtis. La calledrale di Troia, Bénévent. [s.d.]. p. 6.

21. Exode, 12, 7 et 22 . 
inscrire sur le seuil et sur les portes de chaque maison", scribes ea in limine el ostiis domus luae ${ }^{22}$. Les portes du temple élevé par le roi Salomon à Jérusalem seront toutes en or ${ }^{23}$, car elles donnaient accès à "la maison du Seigneur", "la maison du Saint des saints", "la maison de Dieu» ". Ainsi la porta aurea mentionnée par Suger nous conduit-elle à considérer l'église comme le nouveau Temple, la "maison de Dieu» de la Nouvelle Alliance, rapprochement que fait expressément l'inscription qui se trouvait à l'archivolte du portail de la façade occidentale de l'abbaye poitevine des Moreaux, LT FUIT INTROITUS TEMPLI SANCTI SALOMONIS ${ }^{25}$.

Toute une série d'inscriptions, placées sur les voussures, les tympans, ou à proximité des portes souligne que l'église est la "maison de Dieu» et la "porte du ciel». La référence biblique est évidente. En songe Jacob voit une échelle dont le sommet touche le ciel, des anges de Dieu y montent et y descendent. Le Seigneur l'assure qu'il sera toujours avec lui, et Jacob, se réveillant, s'écrie : "Que ce lieu est redoutable! Il n'est autre que la maison de Dieu et la porte du ciel ». Et il se lève, dresse une pierre en stèle et appelle ce lieu Béthel, c'est-à-dire Maison de Dieu (Genèse, 28, 10-22). De nombreuses inscriptions chrétiennes d'Afrique emploient l'expression DOMLS DOMINI, DOMUS DEI pour désigner l'église ${ }^{26}$, et la maison du Seigneur conduit à la demeure céleste, selon la formule d'Eugène de Tolède pour l'église Saint-Félix in Tatanasio reprise par une inscription carolingienne de Belcodène, aujourd'hui conservée au Musée Borély de Marseille, ECCE DOMLS DOMINI QLE DLCIT AD ATRIA CELI ${ }^{27}$. Au début du IX ${ }^{p}$ s. Alcuin reprend la même formule pour la porte de l'église de Saint-Hilaire-le-Grand de Poitiers, Porta domus Domini haec est de regia celi ${ }^{28}$. A l'époque romane, on trouve une expression semblable, au-dessus d'une porte, à la façade occidentale ou sur un bas-côté, à Saint-Pierre-de-l'Isle ${ }^{29}$ et à Béguey ${ }^{30}$ dans l'Ouest de la France, à San Vincenzo de Galliano di Cantù en Italie ${ }^{31}$ :

\section{HAEC EST DOMLS DEI ET PORTA CAELI,}

inspirée du texte de la vision de Jacob, qui est cité au tympan du portail sud de l'église en ruines Saint-Pierre de Genens, VERE NON EST ALIUD NISI DOMLS DEI ET PORTA SANCTA CELI ${ }^{32}$. C'était la même idée qui était reprise à la porte droite de la basilique de Saint-Paul-horsles-murs à Rome :

\section{AEC DOMLS EST DOMINI ET SACRI JANUA REGNI, PROPERATE POPLLI, HAEC DOMLS EST DOMINI ${ }^{33}$.}

L'inscription du tympan de Saint-Pé-de-Bigorre peut ètre jointe au dossier mème si en son début le mot "porte" ne figure pas : EST DOMLS HIC DOMINI, VIA CAELI ... ${ }^{34}$. Parfois l'inscription

22. Deutéronome, 6,9 .

2:3. 'eme livre des Chroniques, 4, 22.

24. Ibid., chapitre 3 et 4.

25. Corpus des inscriptions de la France médiévale. 2. Jépartemenl de la Vienne, éd. Robert Favreav et Jean Michaun, Paris/Poitiers, 1975, p. 9, n*8 (la façade est aujourd'hui à l'Allen Memorial Art Museum d'Oberlin College, Ohio). Le titre de la collection sera désormais abrégé en C.I.F.M.

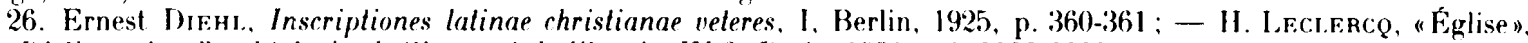
dans Dictionnaire d'archéologie chrétienne et de liturgie, IV-2, Paris, 1921, col. 2222-2223.

27. C.I.F.M. 14. Alpes-Marilimes, Bouches-du-Rhône, Var, éd. R. Favrial, J. Michaud, B. Mora, Paris, Igkg, p. $111-114$

28. Poetae latini aevi carolini, I, ed. Ernest D)trmus.re, Berlin, 1881, p. 326 ("M.G.H., Poetarum latinorum medii aevi*, 1).

29. C.I.F.M. 3. Charente, Charente-Marilime, Deux-Sëures, èd. R. Favrfat et J. Michat:d, Poitiers, 1977, p. 110.

30. C.I.F.M. 5. Dordogne, Gironde, ed. R. Favread, B. Lepi.ant, J. Michald, Poitiers, 1979, p. 86-87.

31. Artur Kingsley Pontik, Lombard Architeclure. II, New Haven/Londres/0xford, 1916, p. 440.

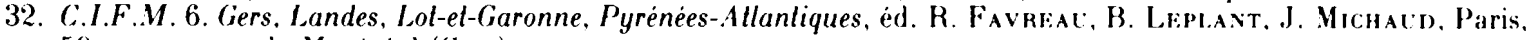
1981, p. 59 : commune de Montréal (Gers).

33. Angelo Suvaciv. Monumenta epigraphica chrisliana saerulo . YIII antiquiora. I. Roma. Pars I .... Rome. I943. pl. XLI, l : l'inscription est aujourd hui dans le cloitre.

34. C.I.F.M. 8. Ariège, Haule-(jaronne, Iautes-Pyrénées, Tarn-el-Garonne, èd. R. Favreat. J. Michald, B. Mora, Paris, 1982, p. 104. 
se borne à assimiler la porte de l'église à la porte du ciel, JANLA COELI, au tympan d'une chapelle en ruines à Malataverne ${ }^{35}$, PORTA POLI, au tympan du portail sud du transept de Saint-Sauveur de Nevers ${ }^{36}$, PORTA CAELI ou COELI à San Juan de la Peña et à Puilampa en Aragon, à Armentia (Alava) ${ }^{37}$, au tympan du portail nord de Thalbürgel en Allemagne ${ }^{38}$.

La source de ces différentes inscriptions est évidente : il s'agit de la liturgie de la dédicace. Le lectionnaire de Luxeuil nous atteste que, dès le $\mathbf{v i I}^{\mathrm{r}} \mathrm{s}$, le texte de la vision de Jacob était lu lors de la cérémonie de dédicace de l'église ${ }^{39}$; et ce même texte est rappelé à propos de la dédicace des églises par le concile d'Aix-la-Chapelle de $836^{40}$. D'après le pontifical romano-germanique du $x^{*}$ s., l'évêque, lors de la cérémonie de dédicace, fait trois fois le tour de l'église en aspergeant les murs, et la troisième fois il dit: Haec est domus Domini ; puis il consacre l'autel, en citant la vision de Jacob ${ }^{41}$. Le répons Haec est domus Domini el porta coeli figure dans la liturgie de dédicace dès les premiers livres liturgiques conservés ${ }^{42}$. Dans la cérémonie de dédicace telle que la décrivent Honorius dit d'Autun ${ }^{43}$ et le Speculum attribué à Hugues de Saint-Victor ${ }^{44}$ le texte même de Jacob à son réveil est à nouveau cité, et dans le Pontifical de Guillaume I)urand au XıI ${ }^{\mathrm{p}} \mathrm{s}$, c'est ce même texte que chante l'évêque lorsqu'il asperge le pavement de l'église ${ }^{45}$. C'est d'ailleurs chez les liturgistes que l'on trouve surtout le commentaire de l'expression "maison de Dieu» appliquée. à l'église : Haec [domus] quoque domus Dei vocalur, quia in ea Dominus adoralur écrit Honorius dit d'Autun ${ }^{46}$, et Sicard de Crémone reprend : Dicitur et domus Dei, quia Deus eam inhabital; unde Jacob : "Hic est domus Dei el porta caeli»" Le lien avec la liturgie est très clairement exprimé dans les inscriptions du portail de la cathédrale d'Esztergom où l'on trouve, au linteau : ATTOLITE PORTAS PRINCIPES VESTRAS, et QUIS EST ISTE REX GLORIAE ${ }^{48}$, c'est-à-dire la question que pose l'évèque, à trois reprises différentes en frappant la porte avec sa crosse, et la réponse que lui fait alors le diacre, avant l'entrée dans l'église au moment de la dédicace, et ce d'après tous les témoins liturgiques conservés. C'est un passage du psaume 24, hymne triomphale pour la translation de l'arche à Sion ${ }^{49}$.

\section{$\stackrel{*}{* *}$}

35. C.I.F.M. 16. Alpes-de-Hautes-Provence, Hautes-Alpes, Ardèche, Drôme, èd. In., à paraitre (Malataverne. Iròme). 36. A. de Surigny, "Le tympan de Saint-Sauveur de Vevers", Bull. monum., XVIII, 1852, p. 32-37; - Marcel Anfray, L'archilecture religieuse du Vivernais au moyen áge. Les églises romanes, Paris, 1951, p. 160); - Jean Duponr, Nivernais-Bourbonnais roman, La Pierre-qui-Vire, 1976, p. 37. Le tympan est conservé au Musée de la Porte du Croux. 37. Aragon roman, par Ángel Canfi.t.as-Lóptze et Angel San Vincente, La Pierre-qui-Vire, 1971, p. 77-7X : PORTA PER HA.VC CAELI FIT PERVIA CIIQLE FIDELI; - Antonio Duran Gudot. "Las inscripciones medievales de la provincia de Huesca». Eslud. de edad media de la corona de Aragón, VIII. 1967, p. 84.

38. Edith Nfubaler, Die romanischen skulptierten Bogenfelder in Sachsen und Thüringen, Berlin. 1972, p. 229-230. pl. 178 ("Corpus der roman. Kunst im Sächsisch-Thüringischen Gebiet," ser. B, 1): - Anton L.egner, Deutsche Kunst der Romanik, Munich, 1982, p. 151.

39. Le lectionnaire de Luxeuil (Paris, ms. lat. 9427), ed. dom Pierre Salomon, Rome, 1944, p. 206-208.

40. Sacrosancta concilia, VII, èd. Ph. LabBe, Paris, 1672, col. 1735.

41. Le pontifical romano-germanique du dixième siècle, éd. Cyrille Vocifo et Reinard Et.ze, Cité du Vatican, I, p. I41 et 144 ("Studi e testin. 226).

42. Corpus antiphonalium officii. IV, ed.dom R.-.J. HEsв: RT, Home. 1970, p. 201. n" 68(K).

43. Gemma animae. 1. I, chap. 168, P.L.. CLXXII, col. 596.

44. Speculum de mysteriis ecclesine, ibid., CLXXVII, col. 338.

45. Le pontifical romain au Moyen Áge, t. III, le Pontifical de Guillaume Murand, ed. Michel Avorise, Cité du Vatican, 1940, p. 472 ("Studi e testi", 88 ).

46. Gemma animae, 1. 1, chap. 227. P.L.. CLXXII, col. 585.

47. Mitrale seu de officiis ecclesiasticis summa, 1. 1, chap. 5, P.L., C.CXIII, col. 26.

18. Isa Ragitsa, "Porla patet vitae, sponsus vocal intro venite and the Inscriptions of the Lost Portal of the Cathedral of Esztergom". Zeitschr. f. Kunsigesch., XI.III, 1980, p. 348.

49. Ps. 24 (23): 7 et 9.8 . 
"Maison de I)ieu " et par là "porte du ciel " ${ }^{50}$, l'église est un lieu de prière et de paix pour les fidèles qui y entrent. Les premières inscriptions chrétiennes disent l'église DOMLS ORATIONIS, daprès les paroles du Christ chassant les vendeurs du Temple : domus mea domus orationis vocabilur (Matthieu, 21, 13). On retrouvera l'expression aussi bien dans la liturgie de dédicace que dans les inscriptions des façades romanes. De mème le pax hic domui, que le Christ recommande aux soixante-douze disciples de dire chaque fois qu'ils entrent dans une maison (Luc, 10,5), est dit par l'évèque lorsqu'il entre dans l'église qu'il va consacrer, et se rencontre dans nombre d'inscriptions ( Nant, Neuville-lès-I)ecize, La Chapelle-(Gonaguet, Vaison-la-Romaine, Mayence, Subiaco, etc.). si la porte n'est pas citée dans ces deux cas, en revanche une série d'épigraphes qui accompagnent la porte annonce la paix pour celui qui va entrer dans l'église pour prier, pour celui ou pour ceux qui entrent et qui sortent, selon le passage du Deutéronome $(28,6)$, Benedictus eris lu ingrediens et egrediens. La vogue du vers léonin riche pour les inscriptions de qualité au XII et au début du XIII" s. donne des expressions épigraphiques identiques ou très proches à plusieurs textes. Ainsi on trouve:

\section{SIT PAX INTRANTI, SIT GRATIA DIGNA PRECANTI}

aussi bien à Villamartin de Sotoscueva en Castille en 1175 qu’à Cingoli en Italie en 1214 et, avec de légères variantes, à Spolète, à Roda ${ }^{51}$, à Dompeter ${ }^{52}$. On citera à part, car le texte est inscrit sur les heurtoirs mêmes de la porte, l'inscription de Saint-Boniface de Freckenhorst (fin XI ${ }^{\mathrm{e}}$-début $\mathrm{XII}$ I"s.).

\section{HAS JANLAS GENTEM CACSA PRECIBLS INGREDIENTEM JHESLS REX REGLM FACIAT CONSCENDERE CAELUM ${ }^{53}$.}

La paix pour qui entre et sort était déjà souhaitée sur un pavement de mosaique de Sidi-Ferruch en Algerie aux premiers siècles chrétiens: PAX INTRANTI ISTAM JANLAM, PAX ET REMEANTI ${ }^{54}$, qui se retrouve, sous la forme d'un vers léonin riche à Marmoutier en Alsace au XII" s. : SIT PAX INTRANTI, PAX SIT SIMLL HINC REMEANTI ${ }^{55}$, et avec des formules proches, à Saint-Apollinaire et à Saint-Blaise à Rome ${ }^{56}$, à Saint-Maximin de Trèves ${ }^{57}$, à SaintLéger-en-Pons ${ }^{58}$. Quelques inscriptions emploient ce souhait au pluriel, mais sans la forme métrique : PAX INTRANTIBLS, PAX EGREDIENTIBL's à Belcodène au Ix ${ }^{e}$ s. ${ }^{59}$, PAX INTRANTIBLS ET REGREDIENTIBCS à Limoges ${ }^{60}$, ou encore ajoutent le salut à la paix, à

5). La place devant la porte de l’eglise sappelle "parvis", qui vient de paradis.

引). R. Favreat, "Fonctions des inscriptions an Moyen Äge". Cahiers civil. médiev. XXXII, 1989, p. 229-230.

52. Robert Wil.. "Repertoire des inscriptions romanes de l'Alsace", Revue d'Alsace, XCVIII, 1959, p. 60-61, fragments du linteau du portail primitif : SlT PAX I.VTRA.VTl et à la fin : PRECA.VTI. Voir aussi du mème. "Complément", Revue disace, (iXII. 19k6, p. 51.

Ђ3. I rsula Mexnf, Die Türzieher des Mittelalters, Berlin, 1981, p. 208-209, n" 11. pl. 28 ("Bronzegeräte des Mittelaltersin, 2).

5.4. F. I)IEHL, op, rit.. I, p. 3600, n" I829.

5). Franz Xaver Krals, Jie christlichen Inschriften der Kheinlande, II, Fribourg-en-Brisgau/I.eipzig. 1894, p. 52, n" 105): - Robert Wit., op. rit., X(:VIII, 1959), p. 64.

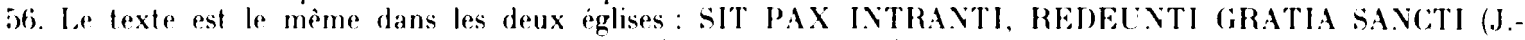
B. de Rossi. Inscriptiones christianae urbis Romae. II. Rome, I888, p. 445): - Mariano Anvel.1.vi, Le chiese di Roma dal secolo IV al XIX, jre ed., Rome, 1891, p. 346.

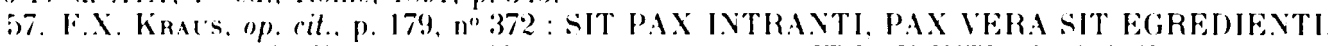

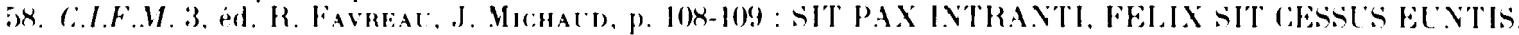

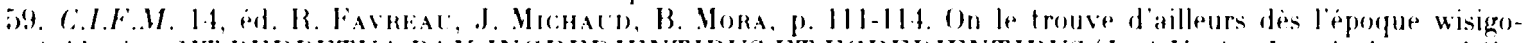

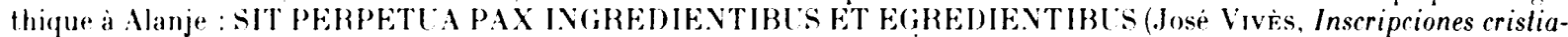
nas de la España romana y risigoda. Barcelone. 1942 et 1969, p. 116, n"33\%).

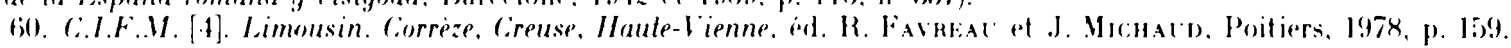


Tauriac ${ }^{61}$ comme à Ĺssin dans la vallée d'Aoste ${ }^{62}$. La versification a conduit à une expression moins ramassée à l'archivolte du portail de Saint-Pierre de Vandeins :

\section{OMNIPOTENS BONITAS EXAUDIAT INGREDIE.NTES A.NGELLS EJLSDEM CLSTODIAT EGREDIENTES ${ }^{63}$.}

On peut penser qu'ici la référence sous-jacente est celle du psaume 121 (120), 8 : Dominus cuslodiat introilum tuum el exilum luum ${ }^{64}$.

La porte de la maison de Dieu est réservée aux justes, selon le texte du psaume 118(117), 20, Haec porta Domini, justi intrabunt in eam qui est de loin le texte biblique le plus fréquemment inscrit sur les églises de Syrie et du Moyen-Orient à l'époque chrétienne ${ }^{65}$. On trouvera ce passage au porche central de la cathédrale de Borgo San Donnino ${ }^{66}$, et il inspire sans doute aussi l'inscription du heurtoir de la porte de l'église abbatiale d'Ebreuil : ADEST PORTA PER QLAM JLSTI REDELNT AD PATRIAM ${ }^{67}$. Le Seigneur garde la porte : DOMINLS EXERCITUM CLSTODIAT HUJLS TEMPLI INTROITUM ET EXITLM, trouve-t-on à la clé de l'arc du tympan à San Pedro de Aguias de Granjinha au Portugal ${ }^{68}$. Il en écarte le mal, comme l'exprime une inscription du $\mathrm{IX}^{\mathrm{e}} \mathrm{s}$. à Oviedo: SIGNUM SALUTIS PONE, DOMINE, IN JANLIS ISTIS, LT NON PERMITTAS INTROIRE A.NGELCM PERCLTIENTEM, qui fait référence aux plaies d'Egypte ${ }^{69}$, ou l'inscription du tympan roman de Saint-Pé-de-Bigorre, où Pierre apparaît comme le gardien : HAEC DATA PORTA PETRO, VADE MALIGNE RETRO ${ }^{70}$. L'accès à la maison de Dieu suppose des sentiments de pénitence, d'où une série importante d'inscriptions qui accompagnent la porte et invitent celui qui va entrer à se corriger. Pour se borner à quelques exemples d'inscriptions de linteaux et tympans au xII" s. on peut citer Saint-Paul-de-Varax (Ain) : "Salut à ceux qui entrent, s'ils mêlent des larmes aux prières. Qu'à leur sortie grâce et pardon comblent ceux qui sortent"; Saint-Rémy-de-Provence : "Qui que tu sois, qui veux entrer en cette demeure sacrée, corrige-toi d'abord avant de pouvoir t'adresser au Christ"; l'église de l'Assomption à Vieu (Ain) : "Ici sans un esprit pur, ni vœux ni dons ne valent. Que ceux qui entrent déposent donc leurs mauvaises pensées" - distique que l'on retrouve presque textuellement à San Adriano de Boñar en Espagne - ; l'ancienne cathédrale Saint-Pierre de Maguelone : "... en passant ces portes, amendez vos mocurs ...". Les inscriptions peuvent aussi se référer au seuil de la porte : "Homme, qui veux te purifier de ton péché, franchis ce seuil ...", au tympan de Saint-Servais de Maastricht, ou, sur les marches de la cathédrale du Puy : "Si tu ne te gardes pas de la faute évite de toucher ce seuil "1". C'est toujours dans le même esprit évangélique, que la porte de la maison de Dieu, ouverte à ceux qui font pénitence, accueille toujours aussi les petits et les pauvres. Le patricien Vilisarius au $\mathrm{XI}^{\circ} \mathrm{s}$. a fondé l'église Santa Maria in Synodo à Rome pour racheter sa faute, et l'inscription qu'il a fait placer à l'extérieur sur une longue plaque de marbre

61. C.I.F.M. 5. Dordogne, Gironde, ed. R. Favread, B. Leplant, J. Michaun, p. 139.

62. X. Barbifin de Montalit, Traile pratique de la construction ...des églises, I, Paris, 1878, p. $51-54$ (Portes).

63. Jean-Claude Col.tex, Les églises romanes de la Hombes, Trévoux, 1978, p. 58-i9.

64. Cette citation se trouve en une quarantaine d'exemples sur des linteaux de portes dans la syrie chretienne (I)enis Frissfi. "L a Bible dans les inscriptions grecques", dans Le monde grec ancien el la Bible, dir. Cl. Moniśssar. Paris. 1984, p. 226 ["Bible de tous les temps", 1]).

65. Ibid., p. 225 (jusqu'à 6 fois dans les églises d'un mème site).

66. Roberto TAssi, II Inomo di Fidenza, Parme, 1973, p. 82 et fig. 47 (HAEG: PORTA I)OMINI, JESTI INTRA.NT PER E.AM).

67. Crsula Mrnof. Die Türzieher des Millelallers, Berlin, I981, p. 214 ("Bronzegeräte des Mittelalters". 2).

68. G... (iraf, Porlugal roman. I. La Pierre-qui-Vire, 1986, p. 249 et fig. 52. La citation du psaume $118(117)$. 20 se retrouve aussi à Biarritz en 1291

69. Juan Enia Ric: "Cuestiones historico-arquelogicas relativas a la ciudad de (Oviedo de los siglos VIII al $\mathrm{X}$ ", dans Symposium sobre cullura asturiana de la alla edad media, Oviedo, 1967, p. 317, note 105, fig. 17.

70. C.I.F.M. 8., ed. R.Favreal, J. Micihal:D, B. Mora, p. 104. Voir Marc, 8,3:3.

71. Références au fichier général du C.I.F.M. ou dans les volumes publies. Pour Maastricht, voir Lisbeth Toi.s. NafrF, La sculpture sur pierre de l'ancien diocèse de liège à l'époque romane, Gembloux, 1957, p. 277-278, pl. 26 B. 
se termine par: "Voici la porte du temple, interdite au seigneur puissant" 72 . Au tympan de Saint-Pé-de-Bigorre "la maison du Seigneur" est dite "l'espoir du pèlerin" "3. Par analogie le chrétien doit toujours garder sa porte ouverte aux pauvres : "Que ta porte soit toujours ouverte aux pauvres, évêque ...", dit un poème de Théodulphe d'Orléans "sur la porte" ${ }^{74}$, "ta porte ne fut jamais fermée aux pauvres" loue l'épitaphe de l'archevêque de Rouen Guillaume en $1110^{75}$, "porte ouverte aux pauvres" dit l'épitaphe de l'archevêque d'Embrun Aimar en $1245^{76}$.

Si la porte a tant d'importance du fait qu'elle donne accès à la "maison du Seigneur", et qu'elle est donc ainsi "porte du ciel», elle mérite encore une plus grande considération parce qu'elle symbolise le Christ lui-même qui s'est dit "la porte des brebis", ego sum ostium ovium (Jean, 10,7), la porte qui ouvre au salut, ego sum ostium. Per me, si quis introerit, salvabitur, et ingredietur et egredielur el pascua inveniel (Jean, 10,9). Les auteurs chrétiens avaient assez peu traité de l'église en elle-même. La plupart des sermons sur la dédicace ont pour thème : ceux qui habitent en la maison de Dieu, ceux-là sont eux-mêmes maison de Dieu ${ }^{77}$. Mais en grand nombre ils ont parlé du Christ qui est la porte par laquelle tout chrétien doit passer. Dans un sermon Augustin enseigne : "le Christ est la porte. Et la porte t'a été ouverte lorsque son côté a été transpercé par la lance" ". Prosper d'Aquitaine écrit que "le Christ est la porte de ceux qui entrent dans le royaume de Dieu" " 79 . Le Christ est devenu la porte, note Paulin de Nole ${ }^{80}$, "Qui peut être appelé la porte sinon le médiateur entre Dieu et les hommes qui a dit: "Je suis la porte" "commente Grégoire le Grand ${ }^{81}$. Uans son commentaire sur l'évangile de Jean, Alcuin développe l'idée d'entrée et de sortie de Jean 10,9: "Que faut-il entendre par entrer et sortir? Entrer dans l'église est bon, sortir de l'église n'est pas bon. Et pourtant on peut entrer bon et sortir bon par la bonne porte qui est le Christ. Celui qui entre par le Christ est digne de louange quand il agit par la foi, celui qui sort par le Christ l'est quand il sort pour cuvrer en bien devant les hommes" ${ }^{82}$. Raban Maur développe tous les sens allégoriques des mots de la Sainte Écriture, mais aussi bien pour janua que pour ostium et porta il donne pour premier sens : "la porte est le Christ" "83. "Le Christ est la porte qui, au nom de la justice, écarte les infidèles de sa maison et qui, par la foi, donne accès aux fidèles" écrit Honorius dit d'Autun ${ }^{84}$. "L'église en laquelle le peuple se réunit pour la louange de Dieu signifie la Sainte Église catholique ... La porte est le Christ ...» dit le Speculum de mysteriis ecclesiae attribué à Hugues de Saint-Victor ${ }^{85}$. Comme Raban Maur, Garnier de Saint-Victor a commenté

72. A. Sillvagni, op. cit., pl. XI,4.

73. On retrouve cette mème idée dans une inscription de l'A frique chretienne: HEC: PORTA DOMLS EST ECRESIF PATE.NS PEREGRINIS ET P[AUPERIBUS]... (E. Dient, op. cit., I, p. 363, no 1844A; - 11. Lecterce, L'Afrique chrétienne, II, Paris, 1904, p. 41).

74. Poetae latini aevi rarolini, I, ed. E. Dу emmi.fr, Berlin, 1881, p. 556.

75. The Ecclesiastical Mistory of Orderic Vitalis, VI, éd. Marjorie Chinnal.,., Oxford, 1978, p. 172.

76. C.I.F.M. 15. La ville de Vienne en Iauphine, éd. H. Favreat, J. Michaud, B. Mora, Paris, 1990, n"141.

77. Augustin, Sermons 336 et 337, P.L., XXXVIII, col. 1471 (domus Dei nos ipsi) et 1477; - Ibid., XI.VI, col. 1101. Haymon d'Halberstadt, ibid., (XXVIII, col. 813. Pierre Damien, ibid.. CXI.IV, col. 910.

78. Ibid., XXXV1II, col.1415; Augustin traite aussi du Christ qui est la porte dans ses commentaires sur saint Jean, ibid., XXXV, col. 1728, et XXXVIII, col. 781 ou sur les psaumes, XXXVII, col. 1102 et 1103.

79. Ibid., LI, col. 373.

80. Ibid., I.XI, col. 309.

81. Ibid., LXXV. col. 731 ; voir aussi (irégoire, P.L., LXXVI, col. 941.

82. Ibid., C. col. 885 .

83. Ibid., CXII, col. 965-9666 (janua). 1014 (ostium), 1031 (porta). Autres commentaires sur les portes dans son De uninerso libri XXII (ibid., CXI, col. 385).

84. Ibid., CL.XXII, col. 587.

85. Ibid., CLXXVII, col. 335--3366. Le traite posterieur a 1180 n'est pas d'llugues (†1141). 
avec virtuosité le sens de porta et d'ostium avec toujours le sens premier de Christ-porte ${ }^{86}$. Sicard de Crémone décrivant les différentes parties de l'église dit aussi que "la porte de l'église est le Christ " ${ }^{87}$. Le texte de Jean 10,9 figure dans la liturgie de la Pentecôte ${ }^{88}$, mais c'est ici principalement aux commentaires des auteurs chrétiens qu'il doit d'avoir eu une fortune certaine dans les inscriptions.

Le terme d'ostium est régulièrement employé dans l'Ancien Testament pour la porte du Tabernacle, et c'est lui qu'a repris saint Jean. Ln ancien bas-relief en bronze doré à Santa Maria della Monterella, cite le premier texte de Jean $(10,7)$ EGO SLM OSTILM ET OVILE OVILM ${ }^{89}$, et sur le linteau roman de Saint-Pourçain-sur-Sioule on trouve aussi EGO SCM HOSTICM OVILM ${ }^{90}$, mais l'inscription se poursuit par la fin de Jean 10,9: PER ME SI QUIS INTROERIT SALVABITLR, ET INGREDIETUR, ET PASCUA INVENIET DICIT DOMINLS, et c'est plutôt ce verset de Jean qui a été retenu dans les inscriptions. À l'archivolte du portail ouest de l'abbatiale d'Alpirsbach, on a inscrit: EGO SUM OSTILM, DICIT DOMINLS, PER ME SI QLIS INTROEIRIT SALVABITLR ${ }^{91}$, ce qui se retrouve sur le livre tenu par le Christ en tête d'un manuscrit ottonien ${ }^{92}$. Sur le livre tenu par le Christ également, au tympan roman de Saint-.Maur de Huy, on lit : EGO SLM OSTILM ${ }^{93}$.

À une des entrées du baptistère de Latran, peut-être au temps du pape Hilaire (461-468) était inscrit :... CONSTANTIS FIDEI JANUA CiHRISTUS ERIT ${ }^{94}$. À la cathédrale de Carinola, au portail latéral de droite, l'évèque Bernard $(\dagger 1109)$ est dit l'auteur des portes, qui méritera, par cette largesse, le royaume CUJLS JANUA CHRISTUS HABETLR ${ }^{95}$, hémistiche qui se retrouve dans l'inscription du tympan de Notre-Dame de Cassan, QLIA JANLA CHRISTLS HABETUR ${ }^{96}$. L'abbé Suger affirme, de son côté, sur la porte de son abbatiale, en 1444 que le "Christ est la vraie porte" ${ }^{97}$. Le mot janua, qui forme un dactyle, se prète plus facilement qu'ostium à la versification, et se trouve employé dans un hexamètre dans les trois dernières inscriptions citées. Le vers inscrit au tympan de Santa Cruz de la Seros au xir ${ }^{\mathrm{e}}$ s., JANUA SLM PERPES PER ME TRANSITE FIDELES, "je suis la porte perpétuelle, passez par moi, fidèles" ${ }^{98}$, rend aussi de façon maladroite le verset 9 du chapitre 10 de Jean. Les contraintes du vers léonin vont conduire $\grave{a}$ des formules très proches sur les tympans du $\mathrm{XII}^{\mathrm{e}} \mathrm{s}$. Le janua vilae qu'emploient Bède, Alcuin, Raban Maur, Marbode ou Baudri de Bourgueil ${ }^{99}$, rime avec VENITE dans l'inscription de la porte du réfectoire d'été à Saint-Maximin de Trèves ${ }^{100}$. Quatre inscriptions de tympans romans

86. Ibid., CXCIII, col. 402-404 et 406-409.

87. Ibid., C:XXIII, col. 21 .

88. Corpus antiphonalium officii, III, éd. R.-J. Hesbrert, p. 196, n'2593.

89. H. Lect.rercQ, "Porte", dans Dictionnaire d'archéologie chretienne el de liturgie, XIV-1, col. 1505.

90. Marcel (ifnermont et Pierre Prader, Allier, Paris, 1938, p. 230 et note 1 (Les églises de France).

91. Tadeusz. Donr7.fniecki, "Majeslas Domini w zbiorach Muzeum Narodowego w Warszawien, Rocznik Muzeum Narodowego w Warszawie, XIX, 1975, p. 226; - Roswitha BüchI.ER, Les tympans romans à personnages des églises de la vallee du Rhin et alentours, et l'influence de l'art roman qu'on y décèle. Poitiers, 1967, thèse doctor. de 3r cycle. p. 19-23 et pl. 26.

92. Die Ottonische Kölner Malerschule. I. Katalog und Tafeln par Peter Bı.осн et Hermann Sc.нxiтzı.:R, Iüsseldorf, 1967. p. 110 et pl. 436 (évangiles d'Abdinghof-Paderborn).

93. L. Tol.teNakne, La sculpture sur pierre de l'ancien diocèse de Liège .... p. 250 et pl. 22 A : - Marie-Louise THE-

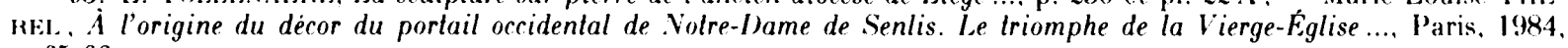
p. 65-66.

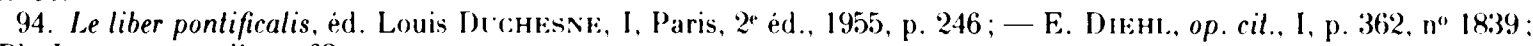
- Ph. Lal'in, op. cil., p. 63.

95. Mario d'ONofro et Valentino PaCE. Campanie romane. p. 123 (il faut lire CLJUS et non CLI, pour le sens et la mètrique).

96. C.I.F.M. 2. Aude, Héraull, éd. H. Favilial, J. Mirhal'W. B. Moha, Paris, 1988, p. 157.

97. Cf. supra, note 19.

98. A. Dirax (il) Dol. Las inscripciones medievales de la provincia de Huesca, p. 105-106.

99. Otto Schl MaNi. Lateinisches Hexameter-Lexikon. Dichlerisches Formelgut von Ennius bis zum Archipoeta, 3. Munich, 1981, p. 8 ("M.G.H., Hilfsmittel", IV/3).

100. F. X. Krals, op. cil.. II, p. 179, no 372 . 
nous offrent la même construction utilisant la rime riche vitae ... venite entre les deux hémistiches. Au portail de San Giorgio in Palazzo de Milan on a :

\section{JANUA SLM VITAE, PRECOR, OMNES INTRO VENITE ${ }^{101}$,}

à Saint-Marcel-lès-Sauzet, dans le Sud-Est de la France, on trouve :

VOS QUI TRA.NSITIS, QLI CRIMINA FLERE VENITIS

PER ME TRANSITE, QUONIAM SUM JANUA VITE, JANLA SLM VITE, VOLO PARCERE, CL[AMO] VENITE ${ }^{102}$.

Le tympan du portail ouest de San Pablo del Campo à Barcelone décline le thème de la porte:

$\div$ HEC DOMINI PORTA, VIA EST OMNIBLS HORTA

JANUA SLM VITE, PER ME GRADIENDO VENITE ${ }^{103}$,

selon une formulation très proche de celle du tympan de Jaxa à Breslau :

JANCA SLM VITE, PER ME QUICLMQLE, VE.NITE ${ }^{104}$.

Sur la fin du $\mathrm{XII}^{\circ}$ s. le narthex peint de l'abbatiale de Payerne a sur le livre tenu par le Christ, un texte de même esprit :

\section{JA.NUA SUM VITE, PER ME MEA REGNA SUBITE ${ }^{105}$.}

Enfin au bord inférieur du linteau du portail de la cathédrale d'Esztergom on pouvait lire :

PORTA PATET VITAE, SPONSUS VOCAT, INTRO VENITE ${ }^{106}$.

Ici l'inscription fait référence à l'Époux qu'attendent les Vierges sages et les Vierges folles (Matthieu 25,1-13), "l'Époux qui est le Christ" comme le dit le jeu liturgique de l'Époux ${ }^{107}$, et au jour du Jugement dernier, comme le suggère une autre inscription du même portail, qui emprunte librement à deux passages de l'Apocalypse $(3,20$ et 19, 17):

\section{FILII, ECCE, PLLSO AD OSTIUM, LT CONGREGEMINI \\ AD ME IN DIEM MAG.NI DEI, LT MANDLCATIS.}

Quiconque vient frapper à cette porte qui est le Christ ne sera pas repoussé. À l'architrave du portail primitif de San Martino in Campo de Monte Albano, on a inscrit la citation presque exacte de Jean 6, 37 : OMNIS QLI VENIT A[D] ME NON EICIAM FORAS ${ }^{108}$, tandis que l'obligation de la métrique a donné une forme approchée à la parole du Christ : "frappez et l'on vous ouvrira" (Matthieu, 7, 7, et 8; Luc 11, 9 et 10) sur le heurtoir de porte de la Johanniterkirche de Würzbourg :

\section{+ INTRA QUI PLLSAS, PETE, NON PACIERE REPULSAS ${ }^{109}$.}

L'antithèse de cette affirmation du Christ "porte de vie», c'est que, désormais, par sa Passion et sa Résurrection la mort est vaincue, et aussi la mort qu'est le péché, comme l'affirment, après le

101. Arthur Kingsley Pontlk, Lombard Archileclure, II, New Haven/Londres/Oxford, 1916, p. 632.

102. C.I.F.M. 16, Drôme, no 66, éd. R. FavrEal, J. Michal,D, B. Moka [à paraitre].

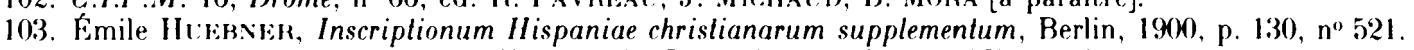

104. Ornamenta ecclesiae. Kunst und Künstler der Romanik, 1, Cologne, 1985, p. 161.

105. Corpus inscriptionum Medii Aevi Ilelvetiae, II, éd. Christoph Jonc; Fribourg, 1984, p. 142-146, no 63, pl. 32-34.

106. Isa Raritsa, op. cil., p. 345-351.

107. Karl Yorwo, The Irama of Medieval Church, II, Oxford, 1933. p. 362, "Sponsus qui est Christus".

108. Guido Monk\%, "Le chiese romaniche del Monte Albano", dans II romanico Pistoiese nei suoi rapporti con l'arte romanica dell' Occidente ..., Pistoia, 1966. p. 38.

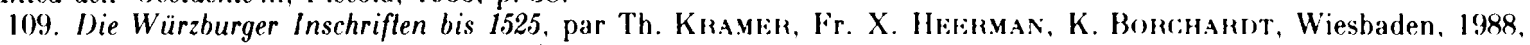
p. 14. $n^{\prime \prime} 17$ ("I)ie deutschen Inschriften", 27). 
psalmiste (Ps. 10 (9), 15) et l'évangéliste (Matthieu, 16, 18), saint Jérôme ${ }^{110}$, Raban Maur ${ }^{111}$ ou Garnier de Saint-Victor ${ }^{112}$. L'inscription du tympan de Peterhausen, du dernier tiers du xil "s. renvoie à ce dernier aspect :

\section{f PRAESIDET HIS PORTIS, QLI SOLVIT VINCLLA MORTIS ${ }^{113}$.}

Le thème de la porte se trouve encore dans les inscriptions relatives à Marie. Litanies mariales ${ }^{114}$, hymnes qualifient la Vierge de janua caeli ou de caeli porta, soulignant d'abord son ròle éminent dans l'Incarnation. C'est ainsi qu'il faut comprendre le tympan de Huy ou le Christ siège en majesté en s'affirmant la porte, EGO SLM OSTIL M, tandis que Marie à sa droite est qualifiée de PORTA COELI ${ }^{115}$. Mais du fait qu'il s'agit de la Maternité divine, les inscriptions ne se situent plus régulièrement à la façade. En la cathédrale de Torcello c'est la mosaïque du cul-de-four qui est consacrée à la "Mère de Dieu", et Marie, à la base du cul-de-four, est dite PORTA SALLTIS ${ }^{116}$, ce qui est le début d'une hymne mariale.

Le texte biblique qui est le plus habituellement appliqué à Marie dans l'épigraphie médiévale est un passage de la description du Temple nouveau par Ezéchiel : "La porte extérieure du sanctuaire, celle qui fait face à l'Orient, ètait fermée. Le Seigneur me dit: "Cette porte restera fermée; on ne l'ouvrira pas; personne n'entrera par là, car le Seigneur, le Dieu d'Israël, est entré par là, elle restera fermée" "(44, 1-2). Ce texte est déjà appliqué à Marie par saint Ambroise" 11 . Raban Maur, dans ses Allégories sur la Sainte Écriture ${ }^{118}$, Geoffroy de Vendôme dans un sermon pour la Nativité ${ }^{119}$, Rupert de Deutz dans son commentaire sur Ezéchiel et son commentaire sur le Cantique des Cantiques ${ }^{120}$. Honorius dit d'Autun dans son Speculum Ecclesiae ${ }^{121}$, Adam de Prémontré dans un sermon de l'Avent ${ }^{122}$, font de même. Une des inscriptions de la chasuble de Saint-Blaise dans l'évêché de Constance utilise le verset 1 du chapitre 44 d'Ezéchiel ${ }^{123}$. Mais c'est de la façon la plus générale le verset 2 qui est cité: + PORTA HEC CLACSA ERIT, NON APERIETUR, ET NON TRANSIBIT PER EAM VIR, QLONIAM DELS ISRAEL INGRESSUS EST PER EAM, dit l'inscription autour de la Vierge dite de Rupert au Musée archéologique de Liège ${ }^{124}$, comme celle de la mosaïque de la chapelle palatine de Palerme ${ }^{125}$, ou, en plus court celle d'une mosaique de Saint-Marc de Venise ${ }^{126}$ et d'une peinture de la chapelle du Liget ${ }^{127}$. On

110. P.L., XXV, col. 1044-1045.

111. Ibid., CXII, col. 1031.

112. Ibid., CXCIII, col. 404.

113. F.X. Krat's, op. cil., II, p. 31-32, n"66.

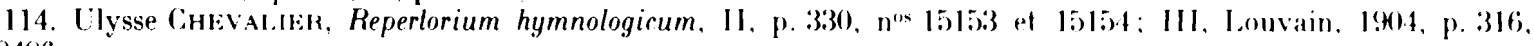
$\mathrm{n}^{\circ} 28486$.

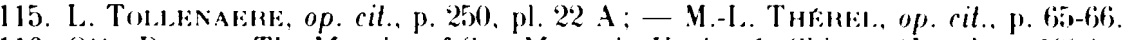

116. Otto Jemis. The Mosaica of San Marco in Venice, 1, Chicago/Londres, 19x.1. p. 40.

117. In apocalypsin expositione. De visione septima. P.I... XVII, col. 9.16.

118. Ibid., CXII, col. 1031 : "Porta, V'irgo .Maria, ut in Ezerhiele: "Porta haes clausa erat. et non aperietur". quod Maria et ante partum incorrupla, el posl partum mansil illaesan.

119. Ibid., CLVII, col. 248.

120. Ibid. CLXVII, col. 1493 ; CLXXIII, col.910.

121. Ibid., CLXXII, col. 905.

122. Ibid.. CXCivitI, col. 186-187.

123. F. X. Kitat's, op. cit, II, p. 42, n" 87

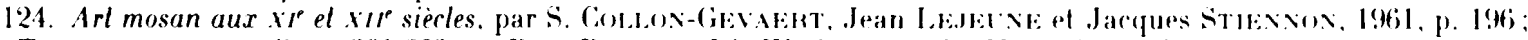
- L. Toldknaknk, op cit. p. 261-262: - Beat Bukik, "Die Werkstätten der Maastrichter Bauplastik des 12. Jahrhunderts", Wallraf-Richartz-Jahrb., XXXVIII, 1976. p. 54.

125. Otto Demi's. The Mosaics of Norman Sicily [en grec], Londres. 1950.

126. II)., The Mosairs of San Marro in V'enice, 2. The thirteenth Cientury, Chicago Londres. 198.4. p. 46.

127. R. Favnear. "Peinture et eppigraphie. La chapelle du Liget", dans Peintures murales romanes ... [Région du Centre], 1988, p. 43 ("Cahiers de l'Inventaire". 15). 
trouve aussi cette citation dans les vitraux du XIII ${ }^{{ }^{p}}$ s. ${ }^{128}$. Ezéchiel est aussi cité sous une forme non littérale : Vidi portam in domo Domini clausam, qui est empruntée à la liturgie de l'Annonciation et de l'Octave de la Nativité ${ }^{129}$ : on trouve ce texte aux cathédrales de Borgo San Donnino, Ferrare, Crémone, Plaisance, et au plafond peint de Saint-Michel d'Hildesheim.

Les saints sont, pour les chrétiens, des intercesseurs naturels auprès de Dieu, et quelques rares textes en font état en les donnant comme des "portes" pour le salut éternel. Un abbé de Solignac demande dans son épitaphe que l'on prie pour lui Marie, "afin que celle qui est appelée la porte du ciel ne lui soit pas fermée" ${ }^{130}$. Suger demande, sur le retable de son abbatiale, que le "grand Denis lui ouvre les portes du paradis" ${ }^{131}$, et un habitant de Brive supplie saint Jacques d'être pour lu "la porte du salut" 132 .

Auteurs de l'Antiquité tardive et auteurs médiévaux ont longuement disserté sur le symbolisme de la porte, en jouant avec toutes les citations de l'Écriture où intervient le mot de porte. Pour Jérôme les portes peuvent être aussi bien de la vie que de la mort, des vertus que du péché, de l'àme croyante que des pécheurs ${ }^{133}$. Pour Paulin de Nole la triple porte de l'église est l'image de la Trinité ${ }^{134}$. Pour Raban Maur et Garnier de Saint-Victor, qui passent en revue tous les sens des mots janua, ostium, porta ${ }^{135}$, la porte est le Christ, la blessure de la lance au côté du crucifié, Marie, ou les cieux, l'entrée du royaume céleste, le jour du jugement, ou la foi, l'intelligence de l'Écriture, la miséricorde, la conscience, l'amour de Dieu et du prochain, la bonne action, le début de la connaissance de Dieu, la crainte de Dieu, l'œuvre de justice, les vertus, ou encore l'Église, les prédicateurs, les auditeurs, les contemplatifs, les saints docteurs, la bouche, les cinq sens, la sortie de la vie, mais aussi les mauvaises ouvres, les désirs de la chair, les mauvaises pensées, les mauvais docteurs, les esprits malins.

Face à cette accumulation de symboles, les inscriptions qui, nombreuses, citent la porte et sont associées à la porte de l'église, mettent l'accent sur deux grands thèmes, qui constituent une ecclésiologie et une christologie. D'abord la porte ouvre sur la "maison de Dieu", et par là est "porte du ciel». La liturgie de la dédicace, qui souligne fortement cette idée, a certainement ici influencé l'épigraphie. Le fidèle est renvoyé à l'église, qui sur terre est la maison de Dieu et la voie vers le salut. "Cette maison est appelée église parce qu'elle signifie la Sainte Église. Qu'elle soit petite ou grande, elle n'en signifie pas moins la Sainte Église que le très noble temple de Salomon ou la cité sainte de Jérusalem» écrit Bruno d'Asti ${ }^{136}$. En second lieu la porte c'est le Christ lui-mème, par qui doivent passer tous ceux qui veulent entrer dans le royaume des cieux, thème johannique longuement repris par tous les commentateurs. L'intercession de Marie ou des saints

128. Cathèdrale Saint-Jean de Lyon; église des Iominicains de Wimpfen am Veckar.

129. Corpus anliphonalium officii, III, éd. R.-J. IIssBint, p. 406, no 4315 , et IV. p. $348, \mathrm{n}^{\circ} 7394$

130. C.I.F.M. [4]. Limousin, éd. R. Favribal et J. Michal'D, p. 224-225.

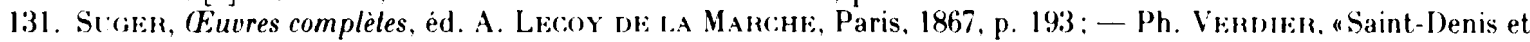
la tradition carolingienne des tituli ....,. p. 346.

132. C.I.F.M. [4]. Limousin ed. R. FavhEat et J. Mir:hat:D, p. 32-33.

133. P.L.. XXV, col. 1044-1045.

134. Ibid., LXI, col. 337, lettre 32 à Sévère : Itaque in ipsi basilicae novae ingressibus hi nersiruli sunt:

Alma domus triplici patel ingredientibus arcui.

Testaturque piam janua trina fidem.

135. Ibid., CXII, col. 965-966, 1014, 1031; CXCIII, col. 402-404, 406-409.

136. Ihid., CL.XV. col. 895. 
est tardive et rare, sauf dans le cas de Marie, pour souligner le mystère de l'Incarnation. De cette double affirmation du Christ et de l'Église la porte tire toute son importance et mérite un décor particulièrement soigné. "Il n'est aucune partie, dans tout l'édifice, qui ne puisse avoir sa propre figure" dit Bruno d'Asti ${ }^{132}$. L'iconographie et ses commentaires épigraphiques peuvent mettre l'accent sur tel ou tel aspect de la porte, mais, même lorsque l'iconographie est absente, on peut toujours penser à cette double valeur de la porte, le Christ, passage obligé pour le fidèle, la "maison de Dieu", l'Église, par laquelle on trouve le chemin du ciel. Les inscriptions qui commentent le thème de la porte nous $\mathrm{y}$ invitent.

* Robert Favreau

C.E.S.C.M. C.I.F.M.

24 , rue de la Chaîne

F - 86022 POITIERS

137. Ibid., col. 896. De figuris ecclesiae : «Si quis autem per singula currere velit, nulla pars in toto edificio reperitur, quae suam non possit habere figuram". 